The Journal of $\mathbf{N}_{\text {onlinear }} \mathbf{S}$ cience and Applications http://www.tjnsa.com

\title{
ON THE DEFINITION OF FUZZY HILBERT SPACES AND ITS APPLICATION
}

\author{
M. GOUDARZI ${ }^{1, *}$ AND S. M. VAEZPOUR ${ }^{1}$ \\ Communicated by D. Mihet
}

\begin{abstract}
In this paper we introduce the notion of fuzzy Hilbert spaces and deduce the fuzzy version of Riesz representation theorem. Also we prove some results in fixed point theory and utilize the results to study the existence and uniqueness of solution of Uryson's integral equation.
\end{abstract}

\section{INTRODUCTION AND PRELIMINARIES}

The notion of probabilistic inner product spaces can be considered as the generalization of that of inner product spaces. The definition of these spaces has been introduced in [3]. Induced norms by these spaces may have very important applications in quantum particle physics particularly in connections with both string and E-infinity theories [6], [7]. Also the definition of a probabilistic Hilbert space, in a special case, has been introduced in [19]. On the other hand, the fuzzy inner product spaces are closely related to the mentioned spaces. Many authors have introduced the definition of fuzzy inner product spaces from different point of views [5], [12], [13]. Recently the definition of a fuzzy inner product space, has been introduced in [10]. A new modification of the last definition can help us to prove the results with less restrictions. On the other hand, how to define the notion of a fuzzy Hilbert space is a quite different story. Creating a structure which be flexible enough to gain the similar results of classical type is among the most important subjects. So, first we modify the definition of a fuzzy inner product space and then we define the notion of a fuzzy Hilbert space. Also we prove some

Date: Received: 2 March 2008; Revised: 15 February 2009.

*Corresponding author.

2000 Mathematics Subject Classification. 46A55; $46 \mathrm{~B} 99$.

Key words and phrases. Fixed point theorem, fuzzy Hilbert space, fuzzy inner product space, Riesz representation theorem, Uryson's integral equation. 
results in fixed point theory and as an application we study the existence and uniqueness of solution of Uryson's integral equation.

Definition 1.1. ([3]) A distribution function(briefly, a d.f.) is a function $F$ from the extended real line $\bar{R}=[-\infty,+\infty]$ into the unit interval $\mathrm{I}=[0,1]$ that is nondecreasing, $F(+\infty)=1, F(-\infty)=0$ and left continuous on $(-\infty,-\infty)$. For every $a \in[-\infty,+\infty]$, the functions $\varepsilon_{a}$ defined by

$$
\varepsilon_{a}(x)= \begin{cases}1 & x \in(a,+\infty] \\ 0 & x \in[-\infty, a] .\end{cases}
$$

are d.f's as is the function $\varepsilon_{\infty}$ defined by $\varepsilon_{\infty}(x)=0$, for all $a \in[-\infty,+\infty)$ and $\varepsilon_{\infty}(+\infty)=1$. The set of all d.f's will be denoted by $\Delta$ and the subset of all d.f's in $\Delta$ satisfying $F(0)=0$ will be denoted by $\Delta^{+}$. For any $\mathrm{F}$ in $\Delta$ we let $\bar{F}$ denote the d.f in $\Delta$ defined via

$$
\bar{F}(x)=l^{-}(1-F(-x)), \forall x \in \mathbb{R},
$$

where $l^{-} F(x)=F\left(x^{-}\right)$. The sets $\Delta$ and $\Delta^{+}$are partially ordered by the usual pointwise partial ordering of functions.

Definition 1.2. ([3]) A triangle function is a binary operation on $\Delta^{+}$that is commutative, associative, non-decreasing in each place and has the $\varepsilon_{0}$ as the identity.

Definition 1.3. ([3]) A multiplication on $\Delta$ is a binary operation $\tau$ on $\Delta$ that is commutative, associative, non-decreasing in each place and whose restriction to $\Delta^{+}$is a triangle function.

A probabilistic inner product(briefly, PIP)space is a quadruple $\left(S, G, \tau, \tau^{*}\right)$, where $\mathrm{S}$ is a real linear space, $\tau$ and $\tau^{*}$ are the multiplications on $\Delta$ such that $\tau \leq \tau^{*}$ and $\mathrm{G}$ is a mapping from $S \times S$ into $\Delta$ such that if $G_{p q}$ denotes the $G(p, q)$ and if the function $\mathcal{N}: S \longrightarrow \Delta^{+}$is defined via

$$
\mathcal{N}_{p}(x)= \begin{cases}G_{p p}\left(x^{2}\right) & x>0 \\ 0 & x \leq 0,\end{cases}
$$

the following conditions hold for all $\mathrm{p}, \mathrm{q}, \mathrm{r}$ in $\mathrm{S}$.

P1a) $G_{p p} \in \Delta^{+}$and $G_{\theta \theta}=\varepsilon_{0}$, where $\theta$ is the null vector in $S$

P1b) $G_{p p} \neq \varepsilon_{0}$ if $p \neq \theta$

P2) $G_{\theta p}=\varepsilon_{0}$

P3) $G_{p q}=G_{q p}$

P4) $G_{-p q}=\bar{G}_{p q}$

P5) $\mathcal{N}_{p+q} \geq \tau\left(\mathcal{N}_{p}, \mathcal{N}_{q}\right)$

P6) $\mathcal{N}_{p+q} \leq \tau^{*}\left(\mathcal{N}_{\alpha p}, \mathcal{N}_{(1-\alpha) q}\right)$, for any $\alpha \in I$

P7) $\tau\left(G_{p r}, G_{q r}\right) \leq G_{(p+q) r} \leq \tau^{*}\left(G_{p r}, G_{q r}\right)$.

Definition 1.4. ([16] and [18]) A binary operation $*:[0,1] \times[0,1] \rightarrow[0,1]$ is said to be a continuous $t$-norm if $([0,1], *)$ is a topological monoid with unit 1 such that $a * b \leq c * d$ whenever $a \leq c$ and $b \leq d(a, b, c, d \in[0,1])$. A t-norm $*$ 
is called strong if it has the two following properties

i) For all $a, b \in(0,1), a * b>0$

ii) For all $a, b, c, d \in[0,1]$ and $a>b, c>d$ we have $a * b>c * d$.

Let $*_{1}, *_{2}$ be two continuous t-norms. We write $*_{1} \geq *_{2}\left(\right.$ resp. $\left.*_{1}>*_{2}\right)$ if $a *_{1} b \geq a *_{2} b$ (resp. $\left.a *_{1} b>a *_{2} b\right)$ for all $a, b \in[0,1]$.

In this paper we denote by $*_{s}$ a strong t-norm. Also in [18, it has been proved that if for all $a, b \in[0,1]$, we define

$$
T_{m}(a, b)=\min \{a, b\} \quad T_{l}(a, b)=\max \{a+b-1,0\} \quad T_{p}(a, b)=a b,
$$

and

$$
T_{d}(a, b)= \begin{cases}\min \{a, b\}, & \text { if } \max \{a, b\}=1 \\ 0, & \text { otherwise, }\end{cases}
$$

Then all of above maps are continuous t-norm and $T_{d}<T_{l}<T_{p}<T_{m}$.

Definition 1.5. ([16]) The 3-tuple $(X, N, *)$ is said to be a fuzzy normed space if $X$ is a vector space, $*$ is a continuous t-norm and $N$ is a fuzzy set on $X \times(0, \infty)$ satisfying the following conditions for every $x, y \in X$ and $s, t>0$

(N1) $N(x, t)>0$

(N2) $N(x, t)=1 \Longleftrightarrow x=0$

(N3) $N(\alpha x, t)=N(x, t /|\alpha|)$ for all $\alpha \neq 0$

(N4) $N(x, t) * N(y, s) \leq N(x+y, t+s)$

(N5) $N(x, \cdot):(0, \infty) \rightarrow[0,1]$ is continuous

(N6) $\lim _{t \rightarrow \infty} N(x, t)=1$.

Example 1.6. ([16]) Let $(X,\|\|$.$) be a normed space. We define a * b=a b$ or $a * b=\min \{a, b\}$ and

$$
N(x, t)=\frac{k t^{n}}{k t^{n}+m\|x\|}, k, m, n \in \mathbb{R}^{+} .
$$

Then $(X, N, *)$ is a fuzzy normed space. In particular if $k=m=n=1$ we have

$$
N(x, t)=\frac{t}{t+\|x\|}
$$

which is called the standard fuzzy norm induced by the norm $\|\cdot\|$.

Definition 1.7. ([17]) Suppose $\left(X, N_{1}, *_{1}\right)$ and $\left(Y, N_{2}, *_{2}\right)$ be two fuzzy normed spaces. A linear operator $T:\left(X, N_{1}, *_{1}\right) \longrightarrow\left(Y, N_{2}, *_{2}\right)$ is said to be fuzzy bounded if there exists a constant $h \in \mathbb{R}^{+}$such that for every $x \in X$ and for each $t>0$

$$
N_{2}(T x, t) \geq N_{1}\left(x, \frac{t}{h}\right)
$$

Theorem 1.8. ([17]) A linear operator $T:\left(X, N_{1}, *_{1}\right) \longrightarrow\left(Y, N_{2}, *_{2}\right)$ is fuzzy bounded if and only if it is continuous.

Proposition 1.9. (15]) Let $(X, M, *)$ be a fuzzy metric space. Then $M$ is a continuous function on $X^{2} \times(0, \infty)$. 
In [10], The following definition has been introduced and then the subsequent results have been proved.

Definition 1.10. A fuzzy inner product space (FIP-space) is a triplet $(X, F, *)$, where $X$ is a real vector space, $*$ is a continuous t-norm and $F$ is a fuzzy set on $X^{2} \times \mathbb{R}$ satisfying the following conditions for every $x, y, z \in X$ and $t \in \mathbb{R}$.

(FI-1) $F(x, y, 0)=0$

(FI-2) $F(x, y, t)=F(y, x, t)$

(FI-3) $F(x, x, t)=H(t) \forall t \in \mathbb{R}$ if and only if $x=0$

(FI-4) For any real number $\alpha$,

$$
F(\alpha x, y, t)=\left\{\begin{array}{lc}
F\left(x, y, \frac{t}{\alpha}\right) & \alpha>0 \\
H(t) & \alpha=0 \\
1-F\left(x, y, \frac{t}{-\alpha}\right) & \alpha<0
\end{array}\right.
$$

$(\mathrm{FI}-5) \sup _{s+r=t}(F(x, z, s) * F(y, z, r))=F(x+y, z, t)$

(FI-6) $F(x, y,):. \mathbb{R} \rightarrow[0,1]$ is continuous on $\mathbb{R} \backslash\{0\}$.

$(\mathrm{FI}-7) \lim _{t \rightarrow+\infty} F(x, y, t)=1$.

Theorem 1.11. Let $(X, F, *)$ be a FIP-space with a continuous t-norm satisfying $t * t \geq t$ for all $t \in[0,1]$. Then it is a (Real) fuzzy normed space (FN-space).

Lemma 1.12. If $(X, F, *)$ is a FIP-space then $F(p, q, t)$ is non decreasing with respect to $t$, for each $p, q \in X$.

Theorem 1.13. (Schwartz inequality) Let $(X, F, *)$ be a FIP-space and $*$ be a continuous $t$-norm with $t * t \geq t$, for all $t \in[0,1]$. Then for any $u, v \in X$ and $t, s>0$ we have

$$
F(u, v, t s) \geq F\left(u, u, t^{2}\right) * F\left(v, v, s^{2}\right) .
$$

Theorem 1.14. (The pythagorean Theorem) Let $(X, F, *)$ be a FIP-space with a continuous t-norm $*$ and $u \perp v$. Then $N(u+v, t)=N(u, t) * N(v, t)$.

Definition 1.15. ([10]) Let $(X, F, *)$ be a FIP-space. $u, v \in X$ is said to be fuzzy orthogonal if $F(u, v, t)=H(t)(\forall t \in \mathbb{R})$ and it is denoted by $u \perp v$.

\section{BASIC RESULtS}

In the first step we give the new modified definition of a fuzzy inner product space and then we prove some interesting results which hold in any fuzzy inner product space. Throughout this paper we let

$$
H(t)= \begin{cases}1 & t>0 \\ 0 & t \leq 0\end{cases}
$$

Definition 2.1. A fuzzy inner product space (FIP-space) is a triplet $(X, F, *)$, where $X$ is a real vector space, $*$ is a continuous t-norm, $F$ is a fuzzy set on $X^{2} \times \mathbb{R}$ and the following conditions hold for every $x, y, z \in X$ and $s, t, r \in \mathbb{R}$.

(FI-1) $F(x, x, 0)=0$ and $F(x, x, t)>0$, for each $t>0$

(FI-2) $F(x, x, t) \neq H(t)$ for some $t \in \mathbb{R}$ if and only if $x \neq 0$ 
(FI-3) $F(x, y, t)=F(y, x, t))$

(FI-4) For any real number $\alpha$,

$$
F(\alpha x, y, t)=\left\{\begin{array}{lc}
F\left(x, y, \frac{t}{\alpha}\right) & \alpha>0 \\
H(t) & \alpha=0 \\
1-F\left(x, y, \frac{t}{-\alpha}\right) & \alpha<0
\end{array}\right.
$$

(FI-5) $F(x, x, t) * F(y, y, s) \leq F(x+y, x+y, t+s)$

$\left(\right.$ FI-6) $\sup _{s+r=t}(F(x, z, s) * F(y, z, r))=F(x+y, z, t)$

(FI-7) $F(x, y,):. \mathbb{R} \rightarrow[0,1]$ is continuous on $\mathbb{R} \backslash\{0\}$

(FI-8) $\lim _{t \rightarrow+\infty} F(x, y, t)=1$.

Example 2.2. Let $(X,<., .>)$ be an ordinary inner product space. We define a mapping $F: X^{2} \times \mathbb{R} \rightarrow[0,1]$ as follows

$$
F(\alpha x, y, t)=\left\{\begin{array}{l}
\frac{k t^{n / 2}}{k t^{n / 2}+m|<\alpha x, y>|^{n / 2}} \quad t>0 \text { and } \alpha \geq 0, \quad k, m, n \in \mathbb{R}^{+}, \\
1-\frac{k t^{n / 2}}{k t^{n / 2}+m|<\alpha x, y>|^{n / 2}} \quad t>0 \text { and } \alpha<0, \\
0 \quad t \leq 0 .
\end{array}\right.
$$

If we define $a * b=\min \{a, b\}$ then $(X, F, *)$ is a FIP-space. In particular if $k=m=n=1$ we have

$$
F(\alpha x, y, t)=\left\{\begin{array}{lc}
\frac{t^{1 / 2}}{t^{1 / 2}+|<\alpha x, y>|^{1 / 2}} & t>0 \text { and } \alpha \geq 0, \\
1-\frac{t^{1 / 2}}{t^{1 / 2}+|<\alpha x, y>|^{1 / 2}} & t>0 \text { and } \alpha<0, \\
0 & t \leq 0,
\end{array}\right.
$$

which is called the standard fuzzy inner product induced by the inner product $<.,{ }^{\prime}>$.

To check the details of above example we consider the case min with the standard fuzzy inner product. The other case can be proved similarly. The conditions (FI-1)-(FI-3) clearly hold. In (FI-4), if $\alpha>0$ then the proof is obvious. In the case in which $\alpha<0$ and $t>0$ we have

$$
\begin{aligned}
F(\alpha x, y, t) & =F(-\alpha(-x), y, t) \\
& =F\left(-x, y, \frac{t}{-\alpha}\right) \\
& =1-\frac{(-t / \alpha)^{1 / 2}}{(-t / \alpha)^{1 / 2}+(|<-x, y>|)^{1 / 2}} \\
& =1-\frac{(-t / \alpha)^{1 / 2}}{(-t / \alpha)^{1 / 2}+(|<x, y>|)^{1 / 2}} \\
& =1-F\left(x, y, \frac{t}{-\alpha}\right) .
\end{aligned}
$$

Since the t-norm min satisfies in $\min _{t \in[0,1]}(t, t) \geq t$, then by theorem 1.11 the condition (FI-5) holds. To prove the condition (FI-6) we note that for any $t \in$ $(0,1)$ there exists unique values $u_{t}$ and $v_{t}$ such that

$$
F\left(x, z, u_{t}\right)=F\left(y, z, v_{t}\right),
$$

and

$$
F\left(x+y, z, u_{t}+v_{t}\right)=\sup _{u+v=u_{t}+v_{t}} \min (F(x, z, u), F(y, z, v))\left(u_{t}+v_{t}\right)=t .
$$

Also the conditions (FI-7) and (FI-8) clearly hold. 
The new definition can help us to restate some results mentioned in [10], by weaker conditions.

Theorem 2.3. Let $(X, F, *)$ be a FIP-space. Then it is a (real) fuzzy normed space.

Proof : Define :

$$
N(x, t)= \begin{cases}F\left(x, x, t^{2}\right), & t>0, \\ 0, & t \leq 0 .\end{cases}
$$

We will show that $N$ satisfies conditions of definition 1.5 In fact conditions N1)N3) are obtained from (FI-1)-(FI-4) and Lemma 1.12 To show N4) assume that $s, t>0$. In this case by (FI-4)-(FI-6) we have

$$
\begin{aligned}
N(x+y, t+s) & =F\left(x+y, x+y,(t+s)^{2}\right) \\
& \geq F\left(x+y, x+y, t^{2}+s^{2}\right) * F(0, x+y, 2 t s) \\
& =F\left(x+y, x+y, t^{2}+s^{2}\right) * 1 \\
& \geq F\left(x, x, t^{2}\right) * F\left(y, y, s^{2}\right) \\
& =N(x, t) * N(y, s) .
\end{aligned}
$$

N5) and N6) follows immediately from (FI-7) and (FI-8).

Also as the results of definition 2.1 and proposition 1.9, we have the following statement.

Proposition 2.4. Let $(X, F, *)$ be a FIP-space. Then $F$ is a continuous function on $X^{2} \times \mathbb{R} \backslash\{0\}$.

Definition 2.5. ([10]) Let $(X, F, *)$ be a FIP-space and $\mathrm{N}$ be its induced fuzzy norm. The sequence $\left\{x_{n}\right\} \subset X$ is called $\tau_{F}$-convergent to $x \in X$ (we write $\left.x_{n} \stackrel{\tau_{F}}{\longrightarrow} x\right)$ if for any given $\varepsilon>0$ and $0<\lambda<1$, there exists a positive integer $N_{0}=N_{0}(\varepsilon, \lambda)$ such that $N\left(x_{n}-x, \varepsilon\right)>1-\lambda$, whenever $n \geq N_{0} .(X, F, *)$ is called $\tau_{F}-$ complete if every $\tau_{F}-$ Cauchy sequence is complete.

Definition 2.6. Let $(X, F, *)$ be a FIP-space. A subset $B$ of $X$ is called fuzzy orthogonal if $x \perp y$, for each $x, y \in B$.

Proposition 2.7. (Generalized Pythagorean Theorem): If $\left\{x_{1}, x_{2}, \ldots, x_{n}\right\}$ is an orthogonal subset of the FIP-space $(X, F, *)$ and $N$ is the induced norm by $F$, then for each $t>0$ :

$$
N\left(\sum_{i=1}^{n} x_{i}, t\right)=N\left(x_{1}, t\right) * N\left(x_{2}, t\right) * \ldots * N\left(x_{n}, t\right) .
$$

Proof : See [10].

Theorem 2.8. (i) Let $(X,\|\|$.$) be an ordinary normed space. Suppose \left\{p_{n}\right\}$ be a sequence in $X$ and consider the topology related to the standard fuzzy norm $N$, induced by the norm $\|$.$\| . Then p_{n} \longrightarrow p$ in $(X,\|\|$.$) if and only if p_{n} \longrightarrow p$ in $(X, N, T)$, for $T=T_{m}$ or $T=T_{P}$ 
(ii) Let $(X, N, T)$ be a fuzzy normed space in which $T \geq T_{l}$. Define $\|\|:. X \longrightarrow$ $[0, \infty)$, for each $p \in X$ and $t>0$ by

$$
\|p\|=\sup \{t: N(p, t) \leq 1-t\} .
$$

Then $(X,\|\|$.$) is an ordinary normed space and p_{n} \longrightarrow p$ in $(X,\|\|$.$) if$ $p_{n} \longrightarrow p$ in $(X, N, T)$.

Proof : see [1].

Corollary 2.9. If $K$ is a nonempty, closed and convex subset of a $\tau_{F}$-complete standard FIP-space $(X, F, \mathrm{~min})$ then there exists a unique vector in $K$ of greatest norm.

Proof : Suppose that for $t>0$

$$
\delta=\sup \{N(x, t): x \in K\}=\sup _{x \in K} \frac{t}{t+\|x\|} .
$$

Then $\delta=\inf _{x \in K}\|x\|$ and hence there exists a sequence $\left\{x_{n}\right\}$ in $K$ such that

$$
\lim _{n \rightarrow \infty}\left\|x_{n}\right\|=\delta
$$

But according to classical version we can prove that $\left\{x_{n}\right\}$ is a Cauchy sequence and $x_{0}=\lim x_{n}=\delta$ is the unique point with the smallest norm, for an element $x_{0} \in K$. So we have

$$
N\left(x_{0}, t\right)=\sup _{x \in K} N(x, t) .
$$

Definition 2.10. If $\mathrm{M}$ is a subset of the FIP-space $(X, F, *)$, then the fuzzy orthogonal complement of $\mathrm{M}$, denoted by $M^{\perp}$, is the set of vectors in $\mathrm{H}$, fuzzy orthogonal to every vector in $\mathrm{M}$.

Lemma 2.11. Let $(X, F, *)$ be a FIP-space. Then $M^{\perp}$ is a closed subspace of $X$, possibly consisting of just the zero vector. However, if $M \neq\{0\}$, then $M^{\perp} \neq X$

Proof : By (FI-4) and (FI-6) we can easily prove that $M^{\perp}$ is a subspace of $X$. To prove the closeness suppose that $\left\{x_{n}\right\}$ be a sequence in $M^{\perp}$ and $\lim _{n \rightarrow \infty} x_{n}=$ $x$. Then by proposition 2.4 for each $y \in M$,

$$
F(x, y, t)=\lim _{n \rightarrow \infty} F\left(x_{n}, y, t\right)=H(t),
$$

on $\mathbb{R} \backslash\{0\}$. By (FI-4), zero is orthogonal to each vector in $X$. Also suppose that $x \perp y$, for each $y \in X$. Then $F(x, x, t)=H(t)$, for all $t \in \mathbb{R}$. Hence $x=0$, by (FI-2) and this completes the proof.

\section{FUZZY VERSION OF RIESZ REPRESENTATION THEOREM}

How we can extend the theory of fuzzy Hilbert spaces parallel to the classical version is the story of this section. First we prove a basic theorem which can help to define the notion of a fuzzy Hilbert space and then we prove the Riesz representation theorem for fuzzy Hilbert spaces. 
Theorem 3.1. Suppose that $(X, F, *)$ be a FIP-space, where $*$ is a strong $t$ norm and for each $x, y \in X, \sup \{t \in \mathbb{R}, F(x, y, t)<1\}<\infty$. Define $<., .>$ : $X \times X \longrightarrow \mathbb{R}$ by

$$
<x, y>=\sup \{t \in \mathbb{R}, F(x, y, t)<1\} .
$$

Then $(X,<., .>)$ is an inner product space.

Proof : : By (FI-3), <.,. $>$ is commutative and by (FI-4), we have $<$ $\alpha x, y>=\alpha<x, y>$, for each $x, y \in X$ and $\alpha \in \mathbb{R}$. By lemma 1.12 and (FI-1), $<$ $x, x>\geq 0$ for each $x, y \in X$. Now suppose that $\sup \{t \in \mathbb{R}, F(x, x, t)<1\}=0$. Then for any $t>0, F(x, x, t)=1$. The nondecreasing property of $\mathrm{F}$ and (FI-2) implies that $x=0$. Conversely suppose that $x=0$. By (FI-4)

$$
\sup \{t \in \mathbb{R}, F(0,0, t)<1\}=\sup \{t \in \mathbb{R}, H(t)<1\}=0 .
$$

Finally we prove that $\langle x+y, z\rangle=\langle x, z\rangle+\langle y, z\rangle$, for each $x, y, z \in X$. By (FI-6), for any $\varepsilon>0$

$$
\begin{aligned}
F(x+y, z, t,<x, z>+<y, z>+\varepsilon) \geq & F\left(x, z,<x, z>+\frac{\varepsilon}{2}\right) \\
& * F\left(y, z,<y, z>+\frac{\varepsilon}{2}\right) \\
= & 1 * 1 \\
= & 1 .
\end{aligned}
$$

This means that

$$
\sup \{t \in \mathbb{R}, F(x+y, z, t)<1\} \leq<x, z>+<y, z>+\varepsilon .
$$

Since $\varepsilon$ is arbitrary it implies that

$$
<x+y, z>\leq<x, z>+<y, z>\text {. }
$$

On the other hand if we get

$$
A=1-\left(1-F\left(x, z,<x, z>-\frac{\varepsilon}{2}\right)\right) *\left(1-F\left(y, z,<y, z>-\frac{\varepsilon}{2}\right)\right),
$$

by (FI-4) and (FI-6) we have

$$
\begin{aligned}
A & =1-F\left(-x, z,<x, z>-\frac{\varepsilon}{2}\right) * F\left(-y, z,<y, z>-\frac{\varepsilon}{2}\right) \\
& \geq F(x+y, z,<x, z>+<y, z>-\varepsilon) .
\end{aligned}
$$

Since $F\left(x, z,<x, z>-\frac{\varepsilon}{2}\right)<1, F\left(y, z,<y, z>-\frac{\varepsilon}{2}\right)<1$ and $*$ is a strong t-norm we have

$$
F(x+y, z,<x, z>+<y, z>-\varepsilon)<1, \forall x, y, z \in X,
$$

and this shows that

$$
\langle x+y, z>\geq<x, z>+<y, z>.
$$

So the proof is complete.

Corollary 3.2. Let $(X, F, *)$ be a FIP-space, where $*$ is a strong t-norm and for each $x, y \in X, \sup \{t \in \mathbb{R}, F(x, y, t)<1\}<\infty$. If we define $\|x\|=<x, x>^{\frac{1}{2}}$, Then $(X,\|\|$.$) is a normed space.$ 
Lemma 3.3. Let $(H,<., .>)$ be a Hilbert space with induced norm $\|.\|_{H}$. Suppose that $(H, F, \min )$ be its induced fuzzy inner product space, where

$$
F(x, y, t)=\frac{t}{t+<x, y>}, \forall x, y \in H, \forall t \in \mathbb{R} .
$$

Then for any sequence $\left\{x_{n}\right\}$ in $H$, the following assertions are equivqlent

i) $\lim _{n \rightarrow \infty}\left\|x_{n}-x\right\|_{H}=0$

ii) $\lim _{n \rightarrow \infty}\left\|x_{n}-x\right\|=0$.

Proof : By definition we have

$$
\begin{aligned}
\left\|x_{n}-x\right\|^{2} & =\sup \left\{t \in \mathbb{R}, \frac{t}{t+<x_{n}-x_{x} x_{n}-x>}<1\right\} \\
& =\max \left\{t \in \mathbb{R}, \frac{t^{2}}{t+<x_{n}-x, x_{n}-x>}\right\} \\
& =<x_{n}-x, x_{n}-x> \\
& =\left\|x_{n}-x\right\|_{H}^{2} .
\end{aligned}
$$

Hence the proof is complete.

According to what we see in above statements, we can define :

Definition 3.4. Let $(X, F, *)$ be a FIP-space, where $*$ is a strong t-norm and for each $x, y \in X, \sup \{t \in \mathbb{R}, F(x, y, t)<1\}<\infty$. Define $<., .>: X \times X \longrightarrow \mathbb{R}$ by

$$
<x, y>=\sup \{t \in \mathbb{R}, F(x, y, t)<1\},
$$

and $\|x\|=<x, x>^{\frac{1}{2}}$ (see theorem 3.1 and corollary 3.2 ). We say that $(X, F, *)$ is a fuzzy Hilbert space if $(X,\|\|$.$) is a complete normed space.$

From now on, we denote by $\left(H, F, *_{s}\right)$ and $\left(F H, F, *_{s}\right)$ any FIP-space having the condition of theorem 3.1 and any fuzzy Hilbert space(FH-space) respectively.

Corollary 3.5. In $\left(H, F, *_{s}\right)$, convergency in $\|$.$\| implies the convergency in \tau_{F}$.

Proof : Suppose that $x_{n} \stackrel{\|\cdot\|}{\longrightarrow} x$. Then

$$
\lim _{n \rightarrow \infty}\left\|x_{n}-x\right\|=0
$$

If we put

$$
t_{n}=\sup \left\{t \in \mathbb{R}: F\left(x_{n}-x, x_{n}-x, t\right)<1\right\},
$$

then for each $\varepsilon>0$ there exists $n_{0} \in \mathbb{N}$ such that $t_{n}<\varepsilon$. But this means that

$$
F\left(x_{n}-x, x_{n}-x, \varepsilon\right)=1, \forall n \geq n_{0},
$$

and hence

$$
\lim _{n \rightarrow \infty} F\left(x_{n}-x, x_{n}-x, \varepsilon\right)=1 .
$$

This implies that $x_{n} \stackrel{\tau_{F}}{\longrightarrow} x$.

Theorem 3.6. (Riesz representation for $\boldsymbol{F H}$-spaces) Let $\left(F H, F, *_{s}\right)$ be a fuzzy Hilbert space. For any $\tau_{F}$-continuous linear functional $f$, there exists a unique element $y \in X$ such that for all $x \in X$ we have

$$
f(x)=\sup \{t \in \mathbb{R}, F(x, y, t)<1\} .
$$


Proof : $f$ is continuous linear functional at $x$ means that $x_{n} \stackrel{\tau_{F}}{\longrightarrow} x$ implies that $f\left(x_{n}\right) \longrightarrow f(x)$, for any sequence $\left\{x_{n}\right\}$ in $X$. If $x_{n} \longrightarrow x$, by corollary 3.5 $x_{n} \stackrel{\tau_{F}}{\longrightarrow} x$. So $f$ is also continuous on $F H$ as a Hilbert space. By using Riesz representation theorem the proof is complete.

\section{FiXeD POINT THEOREMS}

In this section we try to gain some common fixed point theorems in fuzzy Hilbert spaces. But first we prove a result which will be useful in the sequel and holds in any Banach space.

Proposition 4.1. Let $S$ and $T$ be a pair of continuous self mapping on the Banach space $(X,\|\|$.$) satisfying the following condition$

$$
\sum_{n=0}^{\infty}\left\|S^{n} x-T^{n} y\right\|<\infty, \forall x, y \in X .
$$

Then there exists an element $x^{*} \in X$ such that

i) $x^{*}$ is the unique common fixed point of $S$ and $T$.

ii) For any given $x_{0} \in X$, the iterative sequences $\left\{S^{n}\right\}_{n=0}^{\infty}$ and $\left\{T^{n}\right\}_{n=0}^{\infty}$ converge to the same point $x^{*}$.

Proof : For any $x_{0} \in X$ choose $\left\{x_{n}=S^{n} x_{0}\right\}_{n=0}^{\infty}$. Then we have

$$
\begin{aligned}
\left\|x_{k+m}-x_{k}\right\| & \leq \sum_{i=k}^{k+m-1}\left\|x_{i+1}-x_{i}\right\| \\
& \leq \sum_{i=k}^{k+m-1}\left(\left\|S^{i} x_{1}-T^{i} x_{1}\right\|+\left\|T^{i} x_{1}-S^{i} x_{0}\right\|\right) .
\end{aligned}
$$

Hence, as $k \longrightarrow \infty$, we obtain

$$
\lim _{k \rightarrow \infty}\left\|x_{k+m}-x_{k}\right\|=0 .
$$

This shows that $\left\{x_{n}\right\}$ is a Cauchy sequence in $X$. Suppose that $x_{n} \longrightarrow u^{*}$. By continuity of $S, S x_{n} \longrightarrow S u^{*}=u^{*}$.i.e. $u^{*}$ is a fixed point of $S$. Similarly, we can prove that for any given $x_{0} \in X$, the sequence $T^{n} x_{0}$ converges to a point $y^{*} \in X$ and $y^{*}$ is a fixed point of $T$. Now we prove that $x^{*}=y^{*}=u^{*}$. In fact let $\mathrm{G}(\mathrm{S})$ and $\mathrm{G}(\mathrm{T})$ be the sets of all fixed points of $S$ and $T$, respectively. Since $u^{*} \in G(S)$ and $y^{*} \in G(T), G(S)$ and $G(T)$ are not empty. For any $x \in G(S)$ and $y \in G(T)$ it follows from (4.1) that

$$
\sum_{n=0}^{\infty}\|x-y\|=\sum_{n=0}^{\infty}\left\|S^{n} x-T^{n} y\right\|<\infty .
$$

This implies that

$$
\|x-y\|=0, \forall x \in G(S), \forall y \in G(T) .
$$

Hence we have $G(S)=G(T)=\left\{u^{*}\right\}=\left\{y^{*}\right\}$ and this completes the proof.

Corollary 4.2. Let $\left\{T_{i}\right\}_{i=1}^{\infty}$ be a sequence of continuous self mappings on the Banach space $(X,\|\|$.$) . Suppose that for any positive integer i, j$ and $i \neq j$ the following holds,

$$
\sum_{n=0}^{\infty}\left\|T_{i}^{n} x-T_{j}^{n} y\right\|<\infty, \forall x, y \in X .
$$


Then there exists an element $x^{*} \in X$ such that

i) $x^{*}$ is the unique common fixed point of $\left\{T_{i}\right\}_{i=1}^{\infty}$.

ii) For any given $x_{0} \in X$ and for any positive integer $i$, the iterative sequence $\left\{T_{i}^{n} x_{0}\right\}_{n=0}^{\infty}$ converges to the same point $x^{*}$.

Definition 4.3. Let $\left(F H, F, *_{s}\right)$ be a fuzzy Hilbert space and $T$ a self mapping on $\mathrm{X}$. $\mathrm{T}$ is said to be a continuous mapping if $x_{n} \longrightarrow x$ implies that $T x_{n} \longrightarrow T x$.

Theorem 4.4. Let $S$ and $T$ be a pair of self mappings on the fuzzy Hilbert space $\left(F H, F, *_{s}\right)$. Suppose that there exists a constant $c \in(0,1)$ such that

$$
F(S x-T y, S x-T y, t) \geq F\left(x-y, x-y, \frac{t}{c}\right), \forall x, y \in F H \text { and } \forall t \in \mathbb{R} .
$$

Then the conclusions of proposition 4.1 hold.

Proof : From (4.3), for all $x, y \in F H$ and $t \in \mathbb{R}$ we have

$$
\begin{aligned}
\sup \{t \in \mathbb{R}, F(S x-T y, S x-T y, t)<1\} & \leq \sup \left\{t \in \mathbb{R}, F\left(x-y, x-y, \frac{t}{c}\right)<1\right\} \\
& =c \sup \{t \in \mathbb{R}, F(x-y, x-y, t)<1\} .
\end{aligned}
$$

Hence for any $n \in \mathbb{N}$, we have

$\sup \{t \in \mathbb{R}, F(S x-T y, S x-T y, t)<1\} \leq c^{n} \sup \{t \in \mathbb{R}, F(x-y, x-y, t)<1\}$.

Therefore

$$
\begin{aligned}
\sum_{n=0}^{\infty}\left\|S^{n} x-T^{n} y\right\| & \leq \sum_{n=0}^{\infty} c^{\frac{n}{2}}\|x-y\| \\
& <\infty
\end{aligned}
$$

By applying (4.4) and corollary 3.2 we can deduce that

$$
\begin{aligned}
\|T x-T y\| \leq & \|T x-S x\|+\|S x-T y\| \\
\leq & \left(\sup \left\{t \in \mathbb{R}, F\left(x-x, x-x, \frac{t}{c}\right)<1\right\}\right)^{\frac{1}{2}} \\
& +\left(\sup \left\{t \in \mathbb{R}, F\left(x-y, x-y, \frac{t}{c}\right)<1\right\}\right)^{\frac{1}{2}} \\
= & 0+c\|x-y\| .
\end{aligned}
$$

This means that $T$ is a continuous self mapping on $\left(F H, F, *_{s}\right)$. The similar argument shows that $S$ is also a continuous self mapping on $\left(F H, F, *_{s}\right)$. Hence the proof is complete.

Note : In the above theorem the condition of continuity has been removed from the conditions in comparison with the ordinary case, although the condition (4.3) shows that $T$ is $\tau_{F}$-continuous.

Corollary 4.5. Let $S$ and $T$ be a pair of self mappings on $\left(F H, F, *_{s}\right)$ and $\left\{c_{n}\right\}_{n=0}^{\infty}$ be a sequence of positive constants such that $\sum_{n=0}^{\infty} c_{n}^{\frac{1}{2}}<\infty$. Suppose further that for $n=0,1,2, \ldots$

$$
F\left(S^{n} x-T^{n} y, S^{n} x-T^{n} y, t\right) \geq F\left(x-y, x-y, \frac{t}{c_{n}}\right),
$$

for all $x, y \in F H$ and $t \in \mathbb{R}$. Then the conclusions of theorem 4.4 hold. 
Proof : By the same argument mentioned in the previous theorem and (4.5)( for $n=$ 1 ) we can prove that $S$ and $T$ are continuous self mappings on $\left(F H, F, *_{s}\right)$ and also for each $x, y \in F H$ we have

$$
\begin{aligned}
\sum_{n=0}^{\infty}\left\|S^{n} x-T^{n} y\right\| & \leq \sum_{n=0}^{\infty}\left(\sup \left\{t \in \mathbb{R}, F\left(x-y, x-y, \frac{t}{c_{n}}\right)<1\right\}\right)^{\frac{1}{2}} \\
& =\sum_{n=0}^{\infty} c_{n}^{\frac{1}{2}}\|x-y\| \\
& <\infty,
\end{aligned}
$$

and this completes the proof.

\section{Application}

Generally we consider the fuzzy inner product spaces as the extension of inner product spaces(example 2.2). So we expect that a fuzzy Hilbert space be an extension of a Hilbert space. In lemma 3.3, we have shown this fact. On the other hand, it seems that the theory of fuzzy Hilbert space be useful to prove many results in Hilbert spaces. We shall utilize the results of section 4 to study the existence and uniqueness of solution of the following kind of Uryson's integral equations on $L^{2}(G)$

$$
x(s)=y(s)+\int_{G} K(s, t, x(t)) d t,
$$

where $G$ is a bounded closed set of $\mathbb{R}^{n}$ and $L^{2}(G)$ is the real linear space of all Lebesgue square integrable functions.

Theorem 5.1. Suppose that $K(s, t, u)(s, t \in G,-\infty<u<+\infty)$ be separable and $K(s, t, u)=k(s, t) u$. Suppose further that $\int_{G}|k(s, t)|^{2} d s d t<1$. If $T$ is the self mapping on $L^{2}(G)$, defined by

$$
T x(s)=y(s)+\int_{G} K(s, t, x(t)) d t,
$$

and satisfies the following condition

$$
\sum_{n=0}^{\infty}\left\|T^{n} x-T_{u}^{n}\right\|_{L^{2}(G)}<\infty, \forall x(s), u(s) \in L^{2}(G) .
$$

Then $T$ has a unique fixed point $x^{*}(s) \in L^{2}(G)$ which is the unique solution for (5.1), for given $y(s) \in L^{2}(G)$, and for any given $x_{0}(s) \in L^{2}(G)$ the iterative sequence

$$
x_{n+1}(s)=y(s)+\int_{G} K\left(s, t, x_{n}(t)\right) d t \quad(n=0,1,2, \ldots)
$$

converges in norm $\|\cdot\|_{L^{2}(G)}$ to $x^{*}(s)$.

Proof : If we define

$$
F(x, u, t)=\frac{t}{t+\int_{G} x(s) u(s) d s}, \forall t \in \mathbb{R} \text { and } \forall x(s), y(s) \in L^{2}(G),
$$

then a simple calculation and lemma 3.3 shows that $\left(L^{2}(G), F\right.$, min) is a fuzzy Hilbert space and

$$
\|x-u\|=\|x-u\|_{L^{2}(G)}, \forall x, y \in L^{2}(G) .
$$


Also from (5.3) and (5.4), we have

$$
\sum_{n=0}^{\infty}\left\|T^{n} x-T^{n} u\right\|=\sum_{n=0}^{\infty}\left\|T^{n} x-T^{n} u\right\|_{L^{2}(G)}<\infty .
$$

Now all conditions of theorem 4.4 are satisfied with $S=T$. So by theorem 4.4, $T$ has a unique fixed point $x^{*}(s) \in L^{2}(G)$. But this means that $x^{*}(s)$ is the unique solution of (5.1). More addition for any given $x_{0}(s) \in L^{2}(G)$, the iterative sequence

$$
x_{n+1}(s)=y(s)+\int_{G} K\left(s, t, x_{n}(s)\right) d t, \quad(n=0,1,2, \ldots)
$$

converges in norm $\|\cdot\|_{L^{2}(G)}$ to $x^{*}(s)$.

Conclusion : In this paper we tried to introduce a reasonable definition of fuzzy Hilbert spaces, which would be flexible enough to extend. As the extension of Hilbert spaces this notion can help us to present easier proofs for many results in ordinary Hilbert spaces, especially in the area of fixed point theory. Also we proved some results which will be useful in the applied area.

\section{REFERENCES}

1. H. Adibi, Y. J. Cho, D. O'Regan, R. Saadati, Common fixed point theorems in $£$-fuzzy metric spaces, Applied Mathematics and Computation, 182(2006), 820-828.

2. C. Alaca, D. Turkoglu and C. Yildiz, Fixed points in intuitionistic fuzzy meric spaces, Chaos, Solitons and Fractals, 29(2006), 1073-1076. 2

3. C. Alsina, B. Schweizer, C. Sempi and A. Sklar, On the definition of a probabilistic inner product space, Rendiconti di Matematica, Serie VII, 17(1997), 115-127.

4. T. Bag and S. K. Samanta, Finite dimensional fuzzy normed linear spaces, J. Fuzzy. Math., 11(2003), 687-705.

5. A. M. El-Abyad and H. M. El-Hamouly, Fuzzy inner product spaces, Fuzzy Sets and Systems, 44(1991), 309-326.

6. M. El-Naschie, On the uncertainity of Cantorian geometry and two-slit experiment, Chaos, Solitons and Fractals 9(1998), 517-529. 1, 1.1, 1.2, 1.3

7. M. El-Naschie, On the unification of heterotic strings, M theory and $\epsilon^{\infty}$ theory, Chaos, Solitons and Fractals 11(2000), 2397-2408.

8. K. Erich, P. M. Radko and P. Endre, Triangular norms, Dordrecht : Kluwer. ISBN 0-79236416-3. 1

9. A. George and P. Veermani, On some results in fuzzy metric spaces, Fuzzy sets and systems, 64(1994), 395-399. 1 1

10. M. Goudarzi, S. M. Vaezpour and R. Saadati, On the intuitionistic fuzzy inner product spaces, Chaos, Solitons and Fractals (2008), doi :10. 1016/j. Chaos. 2008. 04. 040.

11. S. B. Hosseini, D. O'regan and R. Saadati, Some results on intuitionistic fuzzy spaces, Iranian Journal of Fuzzy Systems, 4(2007), 53-64.

12. J. K. Kohli and R. Kumar, On fuzzy inner product spaces and fuzzy co-inner product spaces, Fuzzy Sets and Systems, 53(1993), 227-232. 
13. J. K. Kohli and R. Kumar, Linear mappings, fuzzy linear spaces, fuzzy inner product spaces and fuzzy co-inner product spaces, Bull Calcutta Math. Soc., 87(1995), 237-246. 1, 1, 1.15. $2,2.5,2$

14. M. A. Krasnoselskii, Topological methods in the theory of nonlinear integral equations, Pergamon press. Oxford. Londan. New York. Paris. (1964).

15. J. Rodríguez-lópez, S. Romaquera, The Hausdorrf fuzzy metric on compact sets, Fuzzy Sets and Systems, 147(2004), 273-283.

16. R. Saadati and S. M. Vaezpour, Some results on fuzzy Banach spaces, J. Appl. Math. and computing, 17(2005), 475-484.

17. R. Saadati, A note on some results on the IF-normed spaces, Chaos, Solitons and Fractals (2007), doi :10. 1016/j. Chaos. 2007. 11. 027. 1 1

18. B. Schweizer and A. Sklar, Probabilistic metric spaces, North Holand series in probability and applied mathematics, 1983.

19. Y. Su, X. Wang and J. Gao, Riesz theorem in probabilistic inner product spaces, Int. Math. Forum., 62(2007), 3073-3078. 1.9

$1.4,1.5,1.6$

${ }^{1}$ Dept. of Math., Amirkabir University of Technology, Hafez Ave., P. O. Box 15914, TEHRAN, IRAN

E-mail address: goudarzi@mail.yu.ac.ir

E-mail address: vaez@aut.ac.ir 